\title{
ON THE ADVANTAGES OF A HIGH SPARK FREQUENCY IN RADIO-TELEGRAPHY.
}

\author{
By L. W. Austin.
}

Since practically all of the long-distance receivers used for radio-telegraphy make use of the telephone for the reception of signals, any circumstance which increases the sensitiveness of the telephone increases the sensitiveness of the receiving apparatus in the same measure.

That the telephone is more sensitive for high frequencies has been noted by a great number of observers, the subject having been especially studied by Lord Rayleigh ${ }^{1}$ and M. Wien. ${ }^{2}$ Their results are of particular value, since they as far as possible made use of sine waves, free from the overtones which introduce grave errors in experiments of this kind. Most of Lord Rayleigh's observations were made on a $7 \mathrm{O}-\mathrm{ohm}$ telephone of an ordinary type, and his main results are given in tabular form below.

\begin{tabular}{|c|c|c|c|c|c|c|c|c|c|}
\hline & 128 & 192 & 256 & 307 & 320 & 384 & 512 & 640 & 768 \\
\hline Least audible current $10^{-8} \mathrm{amp}$. & 2800 & 250 & 83 & 49 & 32 & 15 & 7 & 4.4 & 10 \\
\hline
\end{tabular}

Wien studied a number of different types of telephone, and in his paper discusses at some length the relation between frequency and resistance; inductance, and the natural period of the diaphragm. All of these, together with the sensitiveness of the ear to sounds of different pitch $^{3}$, play a part in the phenomenon. As

${ }^{1}$ Phil. Mag. 38, p. 294; 1894.

2 Ann. d. Physik, 4, p. 450; 1901.

${ }^{3}$ Rayleigh, Phil. Mag. 14, p. 596; 1907. 
for the purposes of our practical problem only the summation of these effects is of interest, I will merely cite Wien's values for a Siemens' telephone of 187 -ohm resistance.

\begin{tabular}{r|r|r|r|r|r|r|r|r|r}
\hline $\begin{array}{r}\text { Least audible current } \\
10^{-8} \text { amp ....................... }\end{array}$ & 6400 & 128 & 256 & 512 & 720 & 1024 & 1500 & 2400 & 4000 \\
\hline
\end{tabular}

Both Wien's results and Lord Rayleigh's show a remarkably rapid increase in sensitiveness up to a frequency of about 500, above which the change is slight, showing small maxima depending on the natural periods of the diaphragms. This high degree of sensitiveness continues, according to Wien, up to a frequency of about 2500 .

It is remarkable in the light of these results that, in the attempts to increase the working range of radio-telegraphy, so little attention has been paid to the advantage of a high spark frequency. One cause of the neglect of this question is apparently the widespread though unfounded belief among commercial workers in wireless telegraphy that the newer types of high-resistance telephone, such as they ordinarily use, do not show any great change of sensitiveness with frequency.

It was therefore thought desirable to carry out a similar investigation on telephones of the type used in radio-telegraphy. For this purpose a pair of Schmidt-Wilckes head telephones of about 800 ohms resistance was chosen for investigation. The Bureau of Standards is supplied with a set of dynamos giving fairly pure sine waves and extending over a range of $60-900$ cycles per second. For this experiment the current of $80-100$ milliamperes was measured on a sensitive hot-wire instrument and was then shunted through noninductive shunts so as to produce a sufficiently small fall of potential over a slide wire of known resistance. From this the necessary emf. to just produce an audible sound in the telephone was taken.

The table and curve in Fig. I give the results of the experiment. The change in volt sensitiveness is seen to be approximately one thousand times between 60 cycles and 900 cycles. I have appended the resistance and inductance at 100 and 900 
cycles which were kindly determined for me by Mr. Curtis of the Bureau of Standards. From these values it would undoubtedly be possible to make some approximate estimate of the currents corresponding to the voltages given in the table, but as the inductance and resistance were determined with several milliamperes, while in this experiment we are dealing with from one microampere to less than a thousandth of a microampere, it did not seem certain that the approximation would be very close.

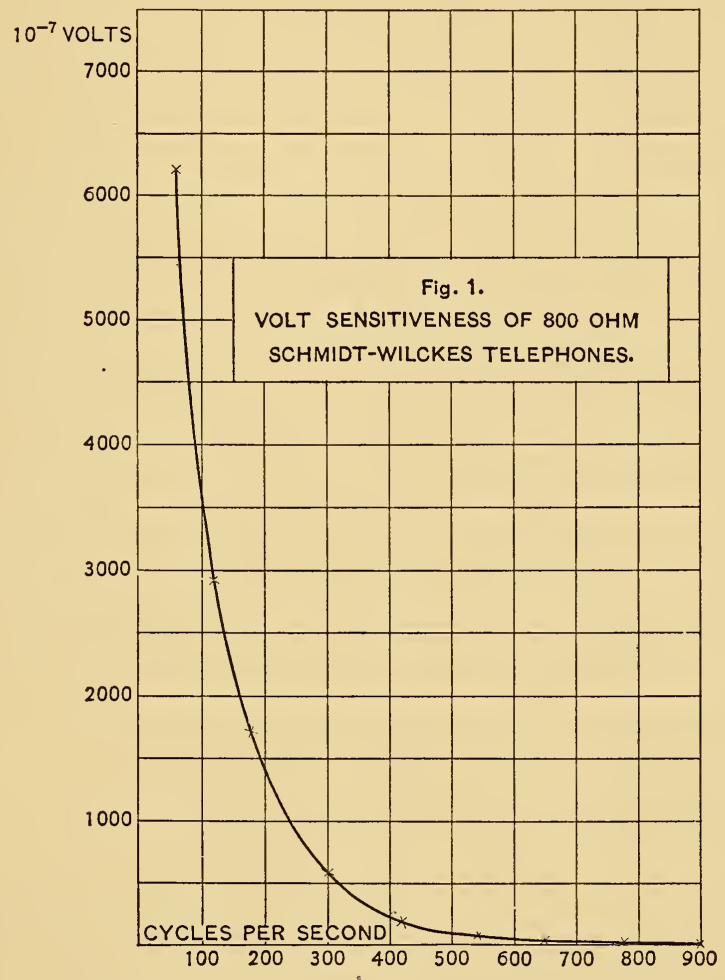

Fig. 1.

Comparing these results with Lord Rayleigh's and Wien's it is seen that the change in sensitiveness is of the same general type, the flat portion of the curve beginning at a somewhat higher frequency, however, and showing no maxima up to the point where the observations ceased. This indicates probably a somewhat higher natural period in the diaphragm. 
Considering these results in their bearing on radio-telegraphy, it appears that we can, by increasing the spark frequency at the sending station, increase the effective sensitiveness of the receiving station many hundred times. This can be done, too, without entailing the difficulties connected with an increase in the sensitiveness of the wireless receiver itself. It is well known that the receivers now in use are already so sensitive that at many stations during the summer months evening receiving is made impossible by atmospheric disturbances. An additional advantage in using a high-pitched musical spark is that the ear picks out such signals with ease in the midst of ordinary interference and atmospheric disturbance. The difficulties of installing apparatus with high spark frequency do not seem to be serious, as I am told that effi-

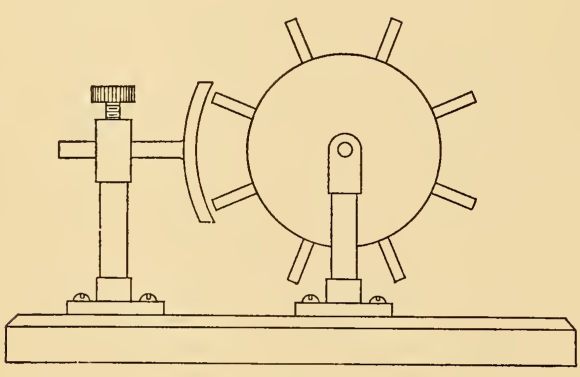

Fig. 2 . cient alternators giving a frequency of 400 , i. e., 800 sparks per second, can be produced without difficulty. The question of cooling the spark gap can, I am sure, be settled by means of some type of rotary gap. A suitable form is shown in Fig. 2. The wheel is rotated rapidly by an independent motor without regard to synchronism with the alternator, the current being conveyed to the wheel by means of brushes. The face of the stationary member of the gap forms an arc of a circle, long enough to a little more than cover the distance between two spokes of the wheel, thus always insuring the proper sparking distance. The rotating wheel itself forms an efficient fan.

In increasing the spark frequency the energy per spark is of course reduced, which is disadvantageous in cases where a receiver of the recording coherer type is used, but experiment shows this reduction in energy per spark to be far more than counterbalanced by the increased sensitiveness of the receiving apparatus, where the telephone is employed.

I have even been able to show in the case of a station using a 6o-cycle alternator and high potential transformer that the increase 
in spark frequency produced by shortening the spark gap and making use of the partial discharges which are usually considered so detrimental, brings about an increase of several times in the loudness of the signals without any increase in power consumed.

There is another quite distinct advantage in spreading a given amount of energy over several sparks instead of concentrating it in one, in that the potential differences are reduced, resulting in a reduction in the condenser losses, which in the average station amount to a considerable share of the total power.

Volt Sensitiveness of a pair of Schmidt-Wilckes 800-ohm Telephones.

\begin{tabular}{c|c}
\hline Cycles. & Volts to produce audible sound. \\
\hline 60 & $6200 \times 10^{-7}$ \\
120 & 2900 " \\
180 & 1700 " \\
300 & 600 " \\
420 & 170 " \\
540 & 80 " \\
660 & 30 " \\
780 & 11 " \\
900 & 6 \\
\hline
\end{tabular}

D. C. Resist. $=8 \mathrm{I} 3$ olims.

$\left.\begin{array}{l}\text { A. C. Resist. }=862 \mathrm{ohms} . \\ \text { Ind. }=404 \mathrm{~m} . \mathrm{h} .\end{array}\right\}$ IOO

A. C. Resist. $=1548$ ohms. Ind. $=24 \mathrm{I} \mathrm{m.} \mathrm{h.}\} 900 \sim$

WAshington, April 27 , I908. $42840-08-$ II 